An. MED. InTERnA (Madrid) Vol. 19, N. $^{\circ} 7$, pp. 331-335, 2002

\section{Mejor resultado del ajuste de la dosis de digoxina en ancianos si se tiene en cuenta que tanto la eliminación como el volumen de distribucion del fármaco disminuyen cuando se reduce la función renal}

\author{
R. CUENA BOY, M. A. ORTIZ DE APODACA RUIZ*,M. A. MACIÁ MARTÍNEZ**
}

Servicio de Medicina Interna. Servicio de Bioquímica Clínica*. Complejo Hospitalario de Toledo. **Centro de Farmacovigilancia de Castilla-La Mancha. Toledo

BEST RESULT OF DIGOXIN DOSING IN THE AGED BY TAKING INTO ACCOUNT THAT BOTH THE ELIMINATION AS WELL AS THE VOLUME OF DISTRIBUTION OF THE DRUG DECREASE WHEN THE KIDNEY FUNCTION DETERIORES

\section{RESUMEN}

Objetivo: Evaluar tres métodos de cálculo de la dosis de digoxina en ancianos.

Métodos: Se calculan las digoxinemias que hubieran alcanzado 87 ancianos ajustando la dosis a la función renal con tres procedimientos diferentes.

Resultados: Edad: 79,0 $\pm 6,3$ años; aclaramiento de creatinina $(\mathrm{Clc})$ : $0,70 \pm 0,23 \mathrm{ml} / \mathrm{Kg}$ de peso magro y minuto. Sólo los métodos que ajustan el aclaramiento de digoxina y su volumen de distribución al Clc consiguen independizar la digoxinemia de la función renal. El mejor entre ellos, consistente en calcular la constante de eliminación (K) y el volumen de distribución (V) como funciones lineales del Clc, de forma que $\mathrm{K}$ oscile entre 0,173 y 0,462 días $^{1}$ y V entre 4 y $101 / \mathrm{Kg}$ de peso magro, respectivamente, cuando el Clc varía de 0 a $110 \mathrm{ml} /$ minuto, obtendría digoxinemias entre 0,8 y $2,0 \mathrm{ng} / \mathrm{ml}$ y superiores a $2,0 \mathrm{ng} / \mathrm{ml}$ en el $81,6 \%$ y el $0,0 \%$ de los pacientes (intervalos de confianza al 95\% (IC 95\%): 72,2\% a 88,4 y $0,0 \%$ a $4,6 \%$ ), respectivamente; con una precisión y un sesgo de 0,43 y $-0,06 \mathrm{ng} / \mathrm{ml}$ (IC 95\%: 0,38 a 0,48 y $-0,16$ a $0,03 \mathrm{ng} / \mathrm{ml}$ ), respectivamente.

Conclusiones: El metodo descrito conseguiría un ajuste inicial de la dosis de digoxina adecuado cuando su indicación no es la reducción rápida de la frecuencia cardiaca en la fibrilación auricular.

PALABRAS CLAVE: Ancianos. Ancianos mayores de 79 años. Digoxina. Monitorización de fármacos. Terapéutica farmacológica.
ABSTRACT

Purpose: To evaluate three methods for digoxin dose adjustment in aged patients.

Methods: We determined the plasma digoxin levels that would be atained in 87 old patients with doses adjusted to the kidney function by means of three separate procedures.

Results: Age: 79,0 "6,3 years of age; creatinin clearance (Clc): 0,70 " $0,23 \mathrm{ml} / \mathrm{Kg}$ of lean body weight and minute. Only the methods that adjust both the digoxin clearance and the volume of distribution to the Clc achieve the independence between the digoxinemia and the kidney function. The best of them, by calculating the elimination constant $(K)$ and the volume of distribution $(V)$ as linear functions of the Clc, so that $K$ ranges between 0,173 and 0,462 days- 1 and $V$ between 4 and $10 \mathrm{l} / \mathrm{Kg}$ of lean body weight when the Clc varies from 0 to $110 \mathrm{ml} /$ minute, achieve digoxinemia figures between 0,8 y 2,0 $\mathrm{ng} / \mathrm{ml}$ and above $2,0 \mathrm{ng} / \mathrm{ml}$ in the $81,6 \%$ and the $0,0 \%$ of the patients $(95 \%$ confidence intervals $(95 \%$ CI): $72,2 \%$ to 88,4 and $0,0 \%$ to $4,6 \%)$, respectively; with a precision and a bias of 0,43 and $-0,06 \mathrm{ng} / \mathrm{ml}$ (95\% CI: 0,38 to 0,48 and $-0,16$ to 0,03 $n g / m l)$, respectively.

Conclussion: The described method would lead to good results if digoxin has not been prescripted in order to control the cardiac fre quency in the setting of auricular fibrilation.

KEY WORDS: Aged. Aged, 80 and over. Digoxin. Drug monitoring. Drug therapy.

Cuena Boy R, Ortiz de Apodaca Ruiz. MA, Maciá Martínez. MA. Mejor resultado del ajuste de la dosis de digoxina en ancianos si se tiene en cuenta que tanto la eliminación como el volumen de distribucion del fármaco disminuyen cuando se reduce la función renal. An Med Interna (Madrid) 2002; 19: 331-335.

\section{INTRODUCCIÓN}

Con digoxinemias $(\mathrm{Cp})$ entre $0,6 \mathrm{ng} / \mathrm{ml}$ y $2,0 \mathrm{ng} / \mathrm{ml}$, en ausencia de circunstancias que modifiquen la sensibilidad al fármaco (hipokaliemia, alteraciones tiroideas, etc.), la mayoría de los pacientes tendrán un efecto terapéutico y será rara la toxicidad grave (1). Por ello, para la elección de la dosis (D) inicial del fármaco interesa disponer de algún sistema que permita alcanzar una $\mathrm{Cp}$ dentro del rango citado en la mayor proporción posible de pacientes.
Entre los diversos factores que modifican la relación $\mathrm{Cp}$ / $\mathrm{D}$ el único controlable cuantitativamente es la función renal. Por ello, todos los autores están de acuerdo en que la dosis de digoxina debe ajustarse al aclaramiento de creatinina $(\mathrm{Clc})$, pero la mayor parte de los métodos existentes para ello tienden a infradosificar a los pacientes (2-6).

En trabajos previos se desarrolló en ancianos un método de ajuste de la D de digoxina al aclaramiento de creatinina (Clc), al que se llamó Lin, que obtuvo mejores resultados que algunos de los métodos publicados anteriormente y que otros

Trabajo aceptado: 4 de abril de 2002

Correspondencia: R. Cuena Boy. Paseo de San Eugenio, 14, 2 B. 45003 Toledo. e-mail: rcuena@hotmail.com 
desarrollados a partir de consideraciones teóricas de índole farmacocinética y sobre información publicada acerca de los parámetros farmacocinéticos de la digoxina $(7,8)$.

Este trabajo evalúa el rendimiento en ancianos del método Lin de dosificación de digoxina y compara su resultado con el que alcanza uno de los sistemas de elección de la dosis inicial de digoxina más empleados, el de Jellife $(2,5)$. Asimismo, se estudia el rendimiento de una modificación del método de Jellife, consistente en tener en cuenta que el volumen de distribución del fármaco (V) depende del Clc (9).

\section{MATERIAL Y MÉTODOS}

Se ha evaluado el rendimiento de tres métodos de ajuste de dosis de digoxina en 87 pacientes ancianos ingresados en el Hospital Geriátrico Virgen del Valle, perteneciente al Complejo Hospitalario de Toledo, que recibían tratamiento oral con digoxina por cualquier indicación y en los que se cumplían las siguientes condiciones: a) La digoxinemia (Cp) se midió a partir de la $7^{a}$ hora posterior a una dosis y tras haber alcanzado el efecto máximo, lo que se produce entre 7 y 20 días con dosis constante (10), dependiendo del Clc; y b) se conocían las características del tratamiento, la talla, el peso, la edad, el sexo y la creatinina sérica de los pacientes y, con excepción de reducciones estables del Clc, no había factores que modificasen la relación entre la D y el Cp. El Clc fue calculado con la fórmula de Cockroft y Gault (11). El cumplimiento terapéutico se supone del $100 \%$ al tratarse de pacientes hospitalizados. Todas las determinaciones de $\mathrm{Cp}$ y de creatinina sérica se realizaron en el Servicio de Bioquímica Clínica del Complejo Hospitalario de Toledo. La medición de la digoxinemia se hizo en todos los casos como parte del control habitual del tratamiento.

Se ha calculado la relación entre la Cp medida, en ng/l, y la dosis (D), en $\mathrm{ng} / \mathrm{Kg}$ de peso magro (PM), que realmente recibe cada paciente (RCpD). El PM se ha hallado mediante el método descrito por Devine (12). Finalmente, se ha estimado la $\mathrm{Cp}$ que se hubiera alcanzado en cada paciente de haberle administrado las D que le corresponderían según cada método de dosificación; para ello se han multiplicado estas D por la $\mathrm{RCpD}$ de cada paciente, y el resultado se ha dividido entre $10^{3}$ a fin de expresar la $\mathrm{Cp}$ en $\mathrm{ng} / \mathrm{ml}$.

Los métodos de dosificación evaluados son los siguientes:

Método Lin: Este sistema se basa en los parámetros farmacocinéticos de la digoxina (9) y en consideraciones farmacocinéticas (13). Según lo publicado, el volumen de distribución (V) de este fármaco disminuye y su vida media de eliminación (t1/2) aumenta al reducirse el Clc, oscilando su rango de valores entre 4 y 10 1/Kg de PM y 1,5 y 4 días, respectivamente (9). Por tanto, y ya que la constante de eliminación (K) es igual al logaritmo neperiano de $2(\ln (2))$ dividido entre la $\mathrm{t} 1 / 2$, la $\mathrm{K}$ de la digoxina varía entre 0,173 y 0,462 días $^{-1}$ al variar el Clc entre 0 y $110 \mathrm{ml} / \mathrm{minuto}$ y se halla con la siguiente ecuación: $K=0,173+0,0026 x \mathrm{Clc}$; en la que el Clc se expresa en $\mathrm{ml} /$ minuto. El V (en $1 / \mathrm{Kg}$ de PM) se calcula con la siguiente ecuación: $V=4+2,84 \times C l c \times 1440 /(P M x 1000)$; en ella el $\mathrm{Clc}$, calculado tambien con el PM, se expresa en $\mathrm{ml} /$ minuto y el PM en $\mathrm{Kg}$ (con esta función V varía entre 4 y 10 1/Kg de PM al aumentar el Clc de 0 a $1,53 \mathrm{ml} /$ minuto y $\mathrm{Kg}$ de PM). El Cld (en 1/Kg de PM y día) se calcula multiplicando K (en días ${ }^{-1}$ ) por $\mathrm{V}$ (en $1 / \mathrm{Kg}$ de $\mathrm{PM}$ ), con lo que se llega a la siguiente función que relaciona el Cld con el Clc: $C l d=0,692+(0,7075 / P M+$
0,0104) $x \mathrm{Clc}+0,01063 \times C l c^{2} / P M$. Teniendo en cuenta que la $\mathrm{Cp}$ en el equilibrio estacionario (Cpee) es igual a la dosis por unidad de tiempo dividida entre el aclaramiento del fármaco, y considerando que en un fármaco en que la fluctuación del nivel plasmático a lo largo del intervalo posológico (en la fase beta) es tan pequeña como en el caso de la digoxina la concentración medida a partir de la $7^{\text {a }}$ hora de una dosis es aproximadamente igual a la Cpee (13), la D teórica diaria intravenosa, en $\mathrm{ng} / \mathrm{Kg}$ de PM, se obtiene multiplicando el Cld calculado por la $\mathrm{Cp}$ deseada de $1200 \mathrm{ng} / \mathrm{l}$, ya que se considera que, salvo existencia de factores predisponentes a la toxicidad digitálica, esta $\mathrm{Cp}$ tiene la mayor posibilidad de ser terapéutica y no tóxica.

Método de Jellife (2): Se halla la fracción de la D de mantenimiento eliminada diariamente (f) con la siguiente fórmula, en la que Clc se expresa en ml/minuto: $f=(4+0,2 \times C l c)$ / 100. La D diaria intravenosa, que es la que repone la cantidad de digoxina eliminada cada 24 horas, se obtiene multiplicando f por una D de carga intravenosa teórica (DI) de $10000 \mathrm{ng} / \mathrm{Kg}$ de PM, dosis habitualmente utilizada cuando es necesaria una digitalización rápida en ancianos (10).

Método de Jellife con V ajustado al Clc (Jellife VA): f se calcula de la misma forma que en el método anterior; sin embargo, en lugar de usar una DI fija, se calcula V con la misma fórmula que en el método Lin y se multiplica por una $\mathrm{Cp}$ deseada de $1200 \mathrm{ng} / \mathrm{l}$ para hallar la DI, en ng/Kg de PM, que se usaría de ser necesaria una digitalización rápida. La $\mathrm{D}$ de mantenimiento diaria intravenosa se obtiene, como en el método Jellife, multiplicando f por la DI.

Las D intravenosas totales diarias en mg se calculan multiplicando las cifras obtenidas con cada método por el PM y dividiendo el resultado entre $10^{6}$. Con los tres métodos las D orales diarias se obtienen dividiendo las intravenosas entre 0,8 ; ya que se supone que la biodisponibilidad de los preparados orales de digoxina es de un $80 \%$ (9).

Para la descripción de las características de los pacientes y su tratamiento se usa la media y la desviación estándar o el porcentaje, según proceda. Se estudia la media, coeficiente de variación (CV), rango y distribución de las Cp obtenidas con cada método de dosificación, con la finalidad de dilucidar con cuál de ellos es mayor el porcentaje de pacientes en que se alcanzan Cp entre 0,8 $\mathrm{ng} / \mathrm{ml}$ y $2,0 \mathrm{ng} / \mathrm{ml}$, valores generalmente terapéuticos y no tóxicos. De este porcentaje y del de Cp superiores a 2,0 ng/ml se calcula el intervalo de confianza al $95 \%$ (IC 95\%). Mediante regresión lineal también se evalúa si las $\mathrm{Cp}$ alcanzadas con los métodos de dosificación siguen dependiendo del Clc. El error (precisión) de cada método de dosificación se evalúa a partir de la raíz de la media de los errores de predicción al cuadrado (rmse) y para el cálculo de la componente de error sistemático (sesgo) se usa el error medio de predicción (me). En ambos casos se hallan los IC 95\%. Asimismo, se comparan los rmse y me de los métodos de Koup y Jellife con los del Lin (14). Tanto el rmse como el me se computan a partir de la diferencia entre la digoxinemia deseada $(1,2 \mathrm{ng} / \mathrm{ml})$ y la que se obtendría en cada paciente con cada método de dosificación.

Teniendo en cuenta los resultados previos del método Lin $(7,8)$, se calculó que con 85 sujetos sería posible calcular el porcentaje de Cp entre 0,8 y $2,0 \mathrm{ng} / \mathrm{ml}$ con un error estándar del $5 \%$ aproximadamente, y que el IC $95 \%$ de las diferencias halladas tanto entre los rmse como entre los me de los métodos de dosificación incluiría el valor cero (error de tipo II) con una probabilidad menor de $0,05 \mathrm{si}$ el valor poblacional de dichas diferencias fuese de $|0,2| \mathrm{ng} / \mathrm{ml}$ o 
más. Por otra parte y para cualquiera de los tres métodos, si el me poblacional fuese $>|0,12| \mathrm{ng} / \mathrm{ml}$ o el rmse poblacional fuese $>0,16 \mathrm{ng} / \mathrm{ml}$, la probabilidad de que el correspondiente intervalo de confianza al $95 \%$ hallado en el estudio incluyese el cero sería menor de 0,05 .

\section{RESULTADOS}

Las características de los pacientes y las de su tratamiento figuran en la tabla I. El resultado de los distintos métodos de ajuste de dosificación se recoge en la tabla II; como puede verse en ella, se alcanzan Cp superiores a 0,7 ng/ml e inferiores a 2,1 $\mathrm{ng} / \mathrm{ml}$ en el 81,6\% (IC 95\%: 72,2\%-88,4\%) de los pacientes con el método Lin, en el 62,1\% (IC 95\%: 51,9\%-72,2\%) con el Jelli $f e$ VA y en el 66,7\% (IC 95\%: 56,2\%-75,7\%) de los casos con el método de Jellife. Los niveles superiores a $2,0 \mathrm{ng} / \mathrm{ml}$ son $0,0 \%$ (IC 95\%: 0,0\%-4,2\%) con Lin y Jellife VA y 6,9\% (IC 95\%: 3,2\%-14,2\%) con el sistema de Jellife.

En la tabla III consta la precisión y el sesgo de los tres métodos, y en la tabla IV la comparación del método Lin con cada uno de los otros dos. Los IC 95\% del sesgo de Lin y Jellife y el de la diferencia entre el me de Lin y de Jellife engloban el valor cero; el IC 95\% del sesgo del sistema Jellife VA y el de la diferencia entre su me y el del Lin no incluyen el 0, por lo que en este aspecto el método Jellife VA es significativamente peor. El IC 95\% de la precisión no incluye el 0 con ninguno de los métodos, por tanto, todos tienen un error significativo, pero el valor de dicho error es menor con Lin y Jellife VA que con Jellife; el IC 95\% de la diferencia entre el rmse de Lin y el del sistema de Jellife no engloba el 0; en consecuencia, desde este punto de vista, Jellife es significativamente peor.

En la figura 1 se representa el nivel obtenido con los métodos de dosificación en función del Clc. No hay asociación lineal estadísticamente significativa entre la $\mathrm{Cp}$ y el Clc con los métodos Lin y Jellife VA. Sin embargo, con el sistema de Jellife existe una asociación lineal inversa y estadísticamente significativa el Clc y la $\mathrm{Cp}$, de forma que son más frecuentes los niveles elevados en pacientes con menor función renal.

\section{DISCUSIÓN}

Con los tres métodos de ajuste de dosis en la mayoría de los pacientes las Cp se encontrarían entre 0,8 y 2,0 ng/ml. Sin
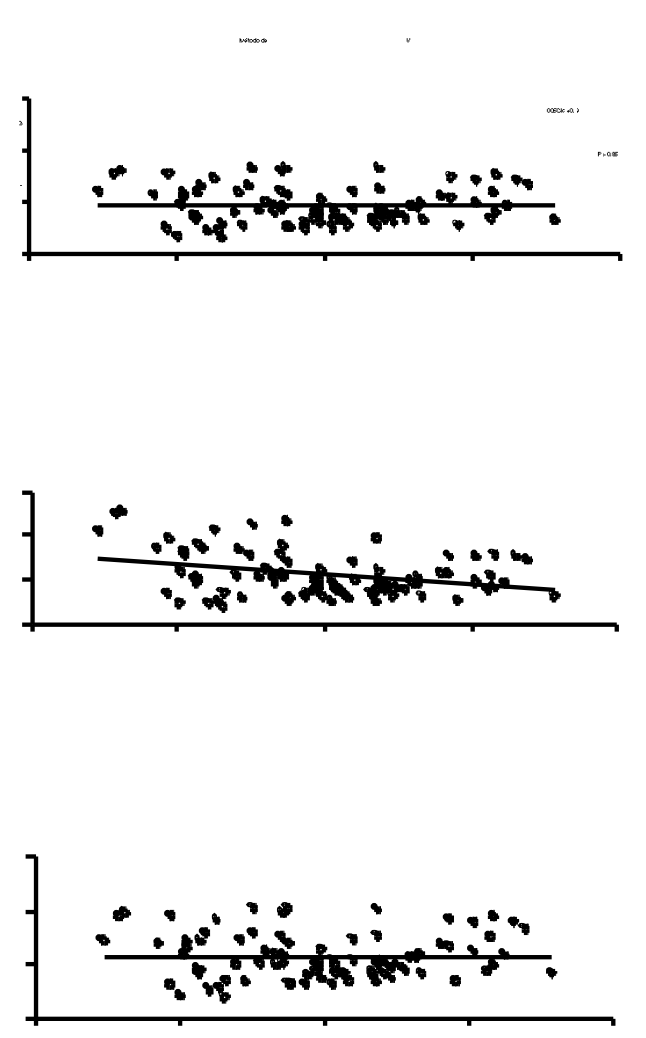

Fig. 1. Relación entre la digoxinemia obtenida con los res métodos y el aclaramiento de creatinina. Abreviaturas: $\mathrm{Cp}$, digoxinemia; $\mathrm{Clc}$, aclaramiento de creatinima.

embargo, el método Lin (cuyas fórmulas figuran a modo de resumen en la tabla $\mathrm{V}$ ) alcanza mejores resultados ya que su menor sesgo, su mayor precisión y su capacidad de independizar $\mathrm{Cp}$ del Clc hacen que en ningún paciente haya niveles claramente supraterapéuticos, a la vez que la media de Cp $(1,1$ $\mathrm{ng} / \mathrm{ml})$ está muy próxima al objetivo $(1,2 \mathrm{ng} / \mathrm{ml})$. Con el método de Jellife, en algún caso podrían alcanzarse Cp próximas a los $3 \mathrm{ng} / \mathrm{ml}$, ello se debe tanto a su menor precisión como a que la $\mathrm{Cp}$ sigue dependiendo del $\mathrm{Clc}$, por lo que las digoxinemias

\section{TABLA I}

\section{CARACTERÍSTICAS DE LOS PACIENTES Y DEL TRATAM IENTO CON DIGOXINA}

\section{Edad (años)}

Peso magro $(\mathrm{Kg})$

$\mathrm{N}$ o y $(\%)$ de mujeres

Creatinina ( $\mathrm{mg} / \mathrm{dl}$ )

Aclaramiento de creatinina ( $\mathrm{ml} / \mathrm{Kg}$ de peso magro y minuto)

Dosis de digoxina ( $\mathrm{ng} / \mathrm{Kg}$ de peso magro y día)

Digoxinemia ( $\mathrm{ng} / \mathrm{ml}$ )

Relación entre digoxinemia y dosis $(\mathrm{Kg} / \mathrm{l})$

Vida media de eliminación de la digoxina (días) ${ }^{\star}$

$$
\begin{gathered}
79,0 \pm 6,3(64-93)^{*} \\
54,3 \pm 10,1(30,6-79,7)^{*} \\
46(52,9 \%) \\
1,3 \pm 0,6(0,7-5,3)^{*} \\
0,70 \pm 0,23(0,14-1,20)^{*} \\
3423 \pm 1345(485-7307)^{*} \\
1,2 \pm 0,5(0,5-2,6)^{*} \\
0,421 \pm 0,237(0,172-1,238)^{*} \\
2,6 \pm 0,36(1,9-3,5)^{*}
\end{gathered}
$$

* M edia \pm desviación estándar y (rango). \&: Hallada a partir de la constante de eliminación calculada como función lineal del aclaramiento de creatinina (método Lin, ver material y métodos para una descripción completa). 
TABLA II

RESULTADO DE LOS M ÉTODOS DE DOSIFICACIÓN

\begin{tabular}{|c|c|c|c|c|c|c|c|}
\hline \multirow[t]{2}{*}{ M étodo } & \multirow{2}{*}{$\begin{array}{c}\text { Digoxinemia } \\
(\mathrm{ng} / \mathrm{l})^{*}\end{array}$} & \multicolumn{5}{|c|}{$\%$ de pacientes según la digoxinemia $(\mathrm{ng} / \mathrm{l}) *$} & \multirow{2}{*}{$\begin{array}{c}\text { Dosis (ng/ } \mathrm{Kg} \text { de } \\
\text { peso magro)* }\end{array}$} \\
\hline & & $<0,6$ & $0,6-0,7$ & $0,8-1,5$ & $1,6-2,0$ & $>2,0$ & \\
\hline $\begin{array}{l}\text { Jellife } \\
\text { VA }^{\star}\end{array}$ & $\begin{array}{c}0,9(38,0 \%) \\
0,3-1,6\end{array}$ & $14,9 \%$ & $23,0 \%$ & $56,3 \%$ & $5,7 \%$ & $0,0 \%$ & $\begin{array}{c}2340(24,9 \%) \\
1095-3596\end{array}$ \\
\hline Jellife ${ }^{£}$ & $\begin{array}{c}1,1(45,6 \%) \\
0,5-2,6\end{array}$ & $8,0 \%$ & $18,4 \%$ & $52,9 \%$ & $13,8 \%$ & $6,9 \%$ & $\begin{array}{c}2742(13,1 \%) \\
1988-3533\end{array}$ \\
\hline $\operatorname{Lin}^{\otimes}$ & $\begin{array}{c}1,1(38,0 \%) \\
0,4-2,0\end{array}$ & $3,4 \%$ & $14,9 \%$ & $63,2 \%$ & $18,4 \%$ & $0,0 \%$ & $\begin{array}{c}2947(25,2 \%) \\
1361-4526\end{array}$ \\
\hline
\end{tabular}

* M edia (coeficiente de variación) y rango. \&: Jellife VA, Jellife y Lin, dosis ajustada con cada uno de los métodos de dosificación (ver material y métodos para una descripción completa de los mísmos).

TABLA III

RENDIM IENTO DE LOS TRES M ÉTODOS DE DOSIFICACIÓN

\begin{tabular}{lccc}
\hline & M étodo Lin & M étodo de Jellife VA & M étodo de Jellife \\
\hline $\begin{array}{l}\text { Precisión: Raiz de la media de } \\
\text { los errores de predicción al cuadrado } \\
\text { (rmse) e intervalo de confianza al 95\% (ng/ } \mathrm{ml})\end{array}$ & $(0,38$ a 0,48$)$ & 0,45 & 0,51 \\
$\begin{array}{l}\text { Sesgo: Error medio de predicción } \\
\text { (me) e intervalo de confianza al }\end{array}$ & $-0,06$ & $(0,41$ a 0,49$)$ & $(0,43$ a 0,58$)$ \\
$95 \%(\mathrm{ng} / \mathrm{ml})$ & $(-0,16$ a 0,03$)$ & $(-0,37$ a- 0,22$)$ & $-0,09$ \\
\hline
\end{tabular}

TABLA IV

RENDIM IENTO DE LOS M ÉTO DOS DE JELLIFE VA Y DE JELLIFE FRENTE AL M ÉTO DO LIN

\begin{tabular}{rrll}
\hline & Método de Jellife VA \\
\hline
\end{tabular}

Precisión relativa: Diferencia entre el rmse del método Lin y

el de los otros dos métodos e intervalo

de confianza al $95 \%$ ( $\mathrm{ng} / \mathrm{ml})$

$-0,02$

$-0,07$

Sesgo relativo: Diferencia entre el me del método Lin y el de los

otros dos métodos e intervalo de

$(-0,06$ a 0,03$)$

$(-0,12$ a $-0,02)$

confianza al $95 \%(\mathrm{ng} / \mathrm{ml})$

0,23

0,03

$(0,21$ a 0,25$)$

$(-0,01$ a 0,07$)$

son mayores cuanto menor es la función renal; esto, precisamente, es lo que se trata de evitar al ajustar la D. Con el sistema Jellife $V A$, debido a su sesgo negativo, serían más frecuentes las infradosificaciones. La capacidad de independizar la $\mathrm{Cp}$ del $\mathrm{Clc}$ que tanto Lin como Jellife VA poseen ilustra la importancia de tener en cuenta los cambios en $\mathrm{V}$, que se producen cuando se modifica la función renal, a la hora de dosificar la digoxina.

El método Lin se ha desarrollado a partir de consideraciones farmacocinéticas teóricas y de información publicada acerca de los parámetros farmacocinéticos de la digoxina, por lo que en la elección del modelo y en su puesta a punto no han intervenido los datos de los pacientes usados para evaluarlo $(7,8)$. Por tanto, el buen resultado alcanzado es base suficiente para emplearlo en la elección de la dosis inicial de digoxina en pacientes ancianos cuando la indicación del fármaco no sea reducir urgentemente la frecuencia cardiaca en una fibrilación auricular y no haya factores predisponentes a la toxicidad digitálica como, por ejemplo, la hipopotasemia; o que modifiquen la relación entre la D y el Cp (exceptuando la edad y las reducciones estables del $\mathrm{Clc}$ ), como interacciones con otros fármacos, hiper o hipotiroidismo, etc. Cuando se pretende un efecto urgente sobre la frecuencia cardiaca, además de plantearse una dosis de carga y de ser precisas con frecuencia Cp superiores a 1,2 ng/ml, la dosis de mantenimiento puede guiarse por la respuesta clínica obtenida; pero incluso en esta situación es útil el método Lin ya que al proporcionar el volumen de distribución facilita el cálculo de las dosis de digitalización.

No obstante, al igual que con cualquier otro método de elección de la $\mathrm{D}$ inicial que tenga la posibilidad de alcanzar un efecto terapéutico en la mayoría de los pacientes, serían necesarias la vigilancia clínica del efecto y analítica de la $\mathrm{Cp}$ mientras se alcanza el equilibrio estacionario. Ello se debe a que la digoxina es un fármaco con rango terapéutico estrecho, con superposición entre las dosis y $\mathrm{Cp}$ tóxicas y terapéuticas, con toxicidad 
potencialmente muy grave que, además, se puede confundir con las manifestaciones propias de la enfermedad que está siendo tratada (por ejemplo, alteraciones del ritmo cardiaco) o ser inespecífica y difícil de detectar (como, por ejemplo, alteraciones sicológicas, especialmente frecuentes en ancianos) y que puede aparecer sin que previamente haya una respuesta terapéutica (10). Así, se ha reportado entre un 13 y un $29 \%$ de incidencia de toxicidad en pacientes hospitalizados que reciben el fármaco $(15,16)$, con una mortalidad del $39 \%$ entre ellos (17). Por tanto, iniciar un tratamiento con digoxina y citar al paciente para revisión al cabo de algunas semanas, sin más controles intermedios, es peligroso, y bien pudiera ser que de esta práctica se deriven fallecimientos atribuidos a la enfermedad de base del paciente y debidos realmente a su tratamiento. En este sentido, otra ventaja del método Lin es que permite el cálculo de la $t 1 / 2$ de eliminación del fármaco (con la fórmula (13):t1/2 = $\ln (2) / K) y$, mediante ella, el del momento aproximado en que se alcanzará el equilibrio y, por tanto, el efecto definitivo.

Otra posibilidad sería comenzar con $\mathrm{D}$ muy pequeñas, menores que las calculadas por los métodos de ajuste de la D al Clc (incluido Lin) y con gran probabilidad de ser infraterapéuticas a la vez que no tóxicas, y, una vez alcanzada la Cpee, evaluar el resultado y hacer las modificaciones necesarias (18). Sin embargo, en los pacientes de este estudio los valores calculados de la $t 1 / 2$ indican que el tiempo que transcurre desde el inicio del tratamiento hasta que se alcanza el equilibrio estacionario (unas cinco veces la $t 1 / 2$ (13)) varía entre 9,5 y 17,5 días, con una media de 13. En muchos casos esto es un periodo demasiado largo para la obtención de un efecto terapéutico, y ello tanto por motivos clínicos como de organización de la asistencia sanitaria, ya que tras cualquier modificación de la D sería necesario volver a esperar el mismo tiempo para observar el efecto definitivo, manteniendo al paciente mientras tanto bajo control. Además, la situación clínica del paciente puede desaconsejar tanto retraso. El sistema Lin permitiría en la mayoría de los pacientes y ya desde el principio administrar una $\mathrm{D}$ terapéutica y sin riesgo inaceptable de toxicidad grave, evitando así un
TABLA V

FÓ RM ULAS DEL M ÉTODO LIN

$\mathrm{PM}=45,5+0,91 \times$ (talla en $\mathrm{cm}-152,4)$; súmense $4,5 \mathrm{Kg}$ en varones.

$\mathrm{Clc}=(140$-edad en años $) \times \mathrm{PM} /(72 \times \mathrm{C})$; multiplíquese
$\quad$ por 0,85 en mujeres.

$\mathrm{K}=0,173+0,0026 \times \mathrm{ClC}$

Tee $=5 \times \ln (2) / K$

$\mathrm{V}=4+2,84 \times \mathrm{ClC} \times 1440 /(1000 \times \mathrm{PM})$

$\mathrm{Cld}=\mathrm{K} \times \mathrm{V}$

$D=1200 \times$ Cld $\times$ PM / 800000

Abreviaturas: $\mathrm{PM}$, peso magro en $\mathrm{Kg}$. Clc, aclaramiento de creatinina en $\mathrm{ml} /$ minuto. $\mathrm{C}$, creatinina sérica en $\mathrm{mg} / \mathrm{dl}$. $\mathrm{K}$, constante de eliminación en días $^{-1}$. Tee, tiempo hasta la acumulación máxima en días. $V$, volumen de distribución en $\mathrm{l} / \mathrm{Kg}$ de peso magro. Cld, aclaramiento de digoxina en //Kg de peso magro y día. D, dosis oral diaria en mg.

número de ajustes de $\mathrm{D}$ y reevaluaciones clínicas y acortando, en consecuencia, el tiempo necesario para llegar a una pauta posológica y un efecto estables. Por otra parte, este método de dosificación, desarrollado específicamente en ancianos, puede impedir que éstos reciban dosis innecesariamente elevadas y, por tanto, reducir la toxicidad sin comprometer la eficacia, ya que las dosis altas en la ancianidad son una de las causas más frecuentes de intoxicación digitálica (19).

\section{FUENTE DE FINANCIACIÓN}

Trabajo financiado en parte con la beca de investigación $n^{\circ}$ 00011/1999 de la Consejería de Sanidad de la Junta de Comunidades de Castilla-La Mancha (España).

\section{Bibliografía}

1. Aronson JK, Hardman M. Digoxin. Br Med J 1992; 305: 1149-52.

2. Jellife RW. An improved method of digoxin therapy. Ann Intern Med 1968; 69: 703-17.

3. Sheiner LB, Benet LZ, Pagliaro, LA. A standard approach to compiling clinical pharmacokinetic data. J Pharmacokinet Biopharm 1981; 9: 59127.

4. Sheiner LB, Rosenberg BG, Marathe VV. Estimation of population characteristics of pharmacokinetic parameters from routine clinical data. $\mathbf{J}$ Pharmacokinet Biopharm 1977; 5: 445-79.

5. Burton ME, Vasko MR, Brater DC. Comparison of drug dosing methods. Clin Pharmacokinet 1985; 10: 1-37.

6. Jones WN, Perrier D, Trinca CE, et al. Evaluation of various methods of digosin dosing. J Clin Pharmacol 1982; 22: 543-50.

7. Cuena Boy R. Evaluación de métodos para la elección de la dosis inicial de digoxina en ancianos. Farm Clín 1998; 15: 10-20.

8. Cuena Boy R. Maciá Martínez MA, López Reneo R, Martín Montero MP. Capacidad predictiva de tres métodos de cálculo de la dosis de mantenimiento de digoxina en ancianos. Arch Cardiol Mex 2001; 71: 306-12.

9. Bresnahan JF. Understanding and using data on serum digoxin concentrations In clinical practice: a case study. En: Moyer TP, Boeckx RL. editores. Applied therapeutic drug monitoring. Vol. 2: review and case studies. Washington: The American Association for Clinical Chemistry, 1984; 97-9.

10. Anónimo. Dosificación de la digoxina. Boletín del Centro Regional de
Farmacovigilancia de Castilla-La Mancha 1995; 6: 10-2.

11. Cockroft DW, Gault MH. Prediction of creatinine clearance from serum creatinine. Nephron 1976; 16: 31-41.

12. Devine B J. Gen-tamicin therapy. Drug Intel Clin Pharm 1974; 8: 650-5.

13. Armijo JA. Leyes generales y aplicaciones de la farmacocinética. En: Flórez J, Armijo A, Mediavilla A. editores. Farmacología humana. $2^{a}$ Ed. Barcelona: Ediciones Científicas y Técnicas, S. A. 1992. p. 75-97.

14. Sheiner LB, Beal SL. Some suggestions for measuring predictive performance. J Pharmacokinet Biopharm 1981; 9: 503-512.

15. Carruthers SG, Kelly JG, McDevitt DG. Plasma digoxin concentrations in patients on admission to hospital. Br Heart J 1974; 36: 707-12.

16. Beller GA, Smith TW, Abelmann WH, Haber E, Hood WB. Digitalis intoxication: a prospective clinical study with serum level correlations. N Engl J Med 1971; 284: 989-97.

17. Fogelman AM, La Mont JT, Finkelstein S, Rado E, Pearce ML. Fallibility of plasma-digoxin in differentiating toxic from non-toxic patients. Lancet 1971; 2: 727-9.

18. Nolan L, Kenny R, O’Malley. The need for reassessment of digoxin prescribing for the elderly. Br J Clin Pharmacol 1989; 27: 367-70.

19. Sánchez de la Rosa R, Rodríguez Hernández N, Cueto Guerreiro T, Rebollo Velázquez A, de Armas Alonso A, Sánchez de la Rosa E. Vigilancia epidemiológica en pacientes tratados con digoxina. Arch Inst Cardiol Mex 1996; 66:510-518 